standing; in such cases we must have recourse to pressure, which has a great effect in removing the tumefaction. Sometimes the disorders occasioned by a wise tooth are still more severe than any that we have as yet described; the following is an example.

CAse V.-M. J., a gentleman about fifty years of age, had suffered excessive torture during the last two years. When I first saw him, the right side of the face was greatly swollen, and disfigured by numerous scars from old abscesses; the neck also was swollen down to the clavicle, and marked in a similar manner; the mouth remained half open and was distorted, the lower teeth not corresponding in range with the upper. The general health of the patient had been considerably affected by his sufferings; for the last four months he laboured under constant, diarrhœa; fetid saliva, mixed with pus, flowed continually from his mouth, and his breath had become so horrible, that he was unable to inhabit the same room with his wife and children. He had passed several months in a maison de Santé, but without benefit. It was in this state that I first saw him, feeble, emaciated, and unable to walk without the support of two friends. A mass of fungous flesh which occupied the whole of the mouth on the diseased side was freely divided, and, after a long examination, a wise tooth was discovered in the base of the coronoid process. The tooth and whole of the process were removed, as were also several portions of the maxillary bone, with loose teeth, \&c. The after-treatment consisted merely in the use of detergent gargles, and it was astonishing to see how rapidly the patient recovered his health and strength. Within a fortnight all bad symptoms had disappeared, and nothing remained but the deviation of the mouth which was finally overcome by means of a bandage

CASE VI.-Chronic inflammation of the amygdala produced and kept up by irregular growth of the wise tooth.

Dr. Friard, while pursuing his medical studies, was attacked, in the summer of 1821 , with pain in the throat, and in the following November with severe inflammation of the right tonsil. This condition was partly subdued by antiphlogistic measures, but the pain \&c. soon returned, and continued, in spite of every means, up to the year 1823. The teeth and gums appeared to be perfectly healthy, and a surgeon was about to extirpate the tonsil, when it was accidentally discovered that the wise tooth on the affected side had not yet made its appearance; the gum was now freely divided, but the portions: of ditided gum became inflamed, and had to be remoted with the knife and caustic. The tooth was, thus completely freed, and the obstinate inflammation of the tofinsil soon disappeared.

This; gentlemen, is the only effectuad treatment whenever the wise tooth is bound down by an indurated portion of gum : you must divide it freely by a deep incision, and it will be right to introduce a small plug of lint between the edges of the wound; the patient may complain of pain, but he must submit, for this is often essential to the success of the operation.

\section{CASE OF RECTO-VAGINAL FISTULA.}

\section{By Dr. VALENTINE MOTT.}

Miss R., 22 years of age, after having travelled from Philadelphia to New York, became affected with abscess in the recto-vaginal region. The abscess pointed in two places: one orifice soon healed up, but the other, situated in the vagina, became fistulous : during four years, injections, caustics, incisions and excisions, in a word, every kind of treatment was employed, but without success. The patient placed herself under my care in March 1839. At this time the fistula opened into the right side of the vagina, about six lines from its entrance. On passing a probe into the fistula, I found that it led upwards to the cavity of the rectum, about three or four inches above the anus. A quantity of air and fæcal matter was occasionally discharged through the opening in the vagina.

It was easy to foresee that the disease should be treated on the same principles as fistula in ano; the cases were analogous, the only difference being, that here the fistula opened into the vagina. However, the ordinary treatment of fistula in ano could not be strictly applied here, for by employing the seton, or dividing the soft parts comprised between the two fistulous openings, we should have destroyed a portion of the perineum, established a large communication between the rectum and vagina, and thus aggravated the disease, instead of curing it.

It therefore became necessary to devise such modifications of the operation for common fistula, as the peculiar nature of this case demanded. Having introduced for several days a large pledget of lint into the fistula, for the purpose of dilating it, and of bringing it into a straight line, I passed a ligature along the fistulous canal (from the vagina) into the rectum, and brought it out through the rectum; it was left in this state for a few days; I then passed the vaginal end of the ligature into the eye of a curved probe, and directed it upwards through the fistulous canal for about an inch and a half. The point of the probe was now turned downwards, and made to strike the perineum, beyond the sphincter ani; I then cut down on the probe, and brought out the end of the ligature. By this means, the ligature, instead of communicating with the rectum at one end, and the vagina at the other, merely embraced (as in cases of common fistula in ano) the inferior portion of the external wall of the rectum, and the soft parts between this latter and the point of incision. Nothing now remained to be done, except to tighten the ligature gradually, in order to destroy the soft parts embraced by it. However, before this was completely accomplished, the vaginatorifice had closed up, and I continued to drat the ligature tighter every day, until the parts embraced by it were reduced to a mere cord, which I cut across + with the scissors. In a few days afterwards the artificial opening healed up, and the patient was perfectly cured of her disease. Twelve months have elapsed since the period of her cure, and I have recently heard from her that she has remained in perfect health ever since.Gaz. Med. No. 18. 